\title{
The Sound of Others: Surprising Evidence of Conformist Behavior th
}

\author{
Paolo Crosetto ${ }^{\mathrm{a}}$, Antonio Filippin ${ }^{\mathrm{b}, \mathrm{c}}$ \\ a INRA and Univ. Grenoble Alpes, UMR 1215 GAEL, F-38000 Grenoble, France \\ ${ }^{b}$ University of Milan, Department of Economics, Via Conservatorio 7, 20122 Milano, Italy \\ cInstitute for the Study of Labor (IZA), Schaumburg-Lippe-Str. 5-9, 53113 Bonn, Germany
}

\begin{abstract}
In this paper we use the "Click" version of the Bomb Risk Elicitation Task to explore preferences for conformism. In the task subjects can infer the behavior of others from the mass of clicks heard. This signal is uninformative about the precise choices of the other participants, and never mentioned in the instructions. We control the exposure of subjects to clicks by implementing treatments with and without earmuffs. We further test the effect of the introduction of a common rather than individual resolution of uncertainty, still keeping individual payoffs independent of other subjects' choices. We find strong evidence of conformist behavior even in such an inhospitable environment. Simply hearing the others clicking marginally affects subjects behavior. Introducing a common random draw results in a dramatic shift of the average choices towards risk loving, in particular by women, which is consistent with social preference considerations.
\end{abstract}

PsycINFO codes: $3020 ; 3040$

JEL Classifications: C81; C91; D81

Word count (excluding references and experimental instructions): 4929

Keywords: Conformism, Risk attitude, Experiment

\section{Introduction}

Conformity has extensively been studied in social psychology since Asch's experiments in the 1950s concerning the desire to fit in a group (Asch, 1951, 1952, 1955, 1956). Conformity has since then been used meaning different things, e.g., the tendency to adhere to a social role (Eagly and Crowley, 1986; Haney et al., 1973a,b), or to social norms (Bicchieri and Xiao, 2009 , among others). In the psychology literature most of the forms of conformist behavior

\footnotetext{
${ }^{\pi}$ We are grateful to the Max Planck Institute in Jena for financial and logistic support and to Florian Strum, Clara Dubois and Denise Hornberger for excellent lab assistance. We thank the participants to the ESA European meeting in Prague for insightful comments. All remaining errors are ours.

Contact: paolo.crosetto@gmail.com (Paolo Crosetto), antonio.filippin@unimi.it (Antonio Filippin)
} 
have been traced back to preferences, i.e., to an intrinsic taste in adapting one's behavior to follow an external reference.

Although there are theories based on preferences (Bernheim, 1994), the empirical literature in economics has mostly focused on the informational content of imitating others: a person may look to others' behavior for guidance when lacking knowledge, absent any specific preference to act like others. Among others, Anderson and Holt (1997); Banerjee (1992); Bikhchandani et al. $(1992,1998)$ built and tested the concepts of herd behavior and information cascades, which show how rational subjects with no conformist preferences might end up disregarding private information and follow the behavior of others. Moreover, since conformity of action might lead to higher correlation in payoffs across subjects, inequality aversion might at least partly rationalize conformist behavior. Bardsley and Sausgruber (2005) use a public good design to show that conformity explains part of the observed crowding-in. Although payoff-irrelevant, the behavior of a different group may still be imitated because of its alleged informational content towards finding one's best reply. Conformist behavior has been empirically observed in choice situations in which subjects are given a private and a public signal, and hence decide on their own or imitate others. Conformist behavior arises even in contexts in which the information content of the public signal is inferior, fuzzier or less grounded than the one emanating from the private signal. ${ }^{1}$ Goeree and Yariv (2015) present an experiment in which subjects must choose between two sources of information: the history of other subjects' past course of actions, that is strictly uninformative, and an informative private signal. Goeree and Yariv (2015) show that a large fraction of subjects still choose to observe the public rather than the private signal. Since in their experiment subjects choose an uninformative public signal, the results of Goeree and Yariv (2015) lend support to the explanation of conformity based on preferences. Thöni and Gächter (2015) interpret the results of a gift exchange game as consistent with conformity driven by preferences. Conformist behavior is observed even more frequently under a common fate condition, in which the choice is not individual, but is rather decided by majority rule, determining equal payoffs for all the participants.

This paper adds to the literature investigating whether a conformist behavior is observed even when the source of information is not pointed out to subjects and possibly not even

\footnotetext{
${ }^{1}$ See the experimental evidence of, among others, Anderson and Holt (1997); Hung and Plott (2001); Quiamzade and L'Huillier (2009); Ziegelmeyer et al. (2010); Corazzini and Greiner (2007) report instead evidence of no conformity.
} 
consciously perceived as a signal by them. We administer to the subjects the Bomb Risk Elicitation Task (Crosetto and Filippin, 2013), an individual risk elicitation measure. Participants see a screen composed of 100 boxes and must collect them by clicking once per box. They earn money for each collected box but lose all earnings in case they collect a bomb that is hidden in one of the boxes and programmed to explode at the end of the task, i.e., after choices have been made. The task is performed and paid in isolated cubicles, with no interaction with the other participants in the experiment other than sitting in the same room and hearing the others click. The noise generated by the other participants constitutes an extremely imprecise proxy of the other subjects' choice and we never mention it in the experimental instructions. This approach is different from almost all previous studies in the economics literature, in which the other subjects' decisions, informative or not, are explicitly shown to the subjects, or at least explicitly presented as being accessible. ${ }^{2}$

We start from the assumption that conformity cannot emerge in a perfectly isolated society. In the Isolated condition we make subjects wear acoustic earmuffs that shield participants from the noise generated by the clicks. The position of the bomb is determined by random draw at the individual level, so that the subjects are in all respects isolated in their cubicles, and the only thing they have in common is sitting in the same room. In the Idiosyncratic Risk treatment we simply remove the earmuffs, everything else being the same, in particular the resolution of uncertainty at the individual level. In the Correlated Risk treatment we introduce a weak form of commonality by imposing the resolution of uncertainty at the aggregate level, i.e. by drawing the same position of the bomb for everyone. This manipulation allows us to ascertain the role of social concerns as a motivation for conformist behavior.

The design of the experiment can be informative from two points of view.

First, it allows us to investigate whether conformity emerges even in an inhospitable environment where subjects do not interact and are not made aware of the existence of the signal. By manipulating the resolution of uncertainty at the individual vs. aggregate level we also analyze whether fuzzy social comparisons play a role in the appearance of conformism. Note in fact that, as explained in detail in Section 2, in the Correlated Risk treatment the payoffs are determined at the individual level. Subjects are paid according to

\footnotetext{
${ }^{2}$ Studying conformist behavior in cheating Fosgaard et al. (2013) show that subjects' behavior is affected both by the awareness that cheating is an option and by knowing that other participants cheated.
} 
how their individual choice compares to the common random event, but by making similar decisions they can ensure similar payoffs, too. In contrast, in the Idiosyncratic Risk treatment, by making the same choice subjects can at most choose the same lottery ex ante, but with no guarantee of similar payoffs as the individual resolution of uncertainty may still lead to opposite outcomes.

Second, the evidence that hearing the clicks of the other participants affects the decisions would constitute a methodological contribution, suggesting that experimental procedures should take it into account in particular when the amount of clicks is substantial and the timing of the choice relevant.

Results lend strong support to the existence of conformist behavior. Subjects tend to follow the unobtrusive signal already in the Idiosyncratic Risk treatment. Despite the absence of any interaction or interdependence between subjects, the average number of boxes collected is higher than in the baseline condition, although the difference is only weakly significant. The small commonality implied by the introduction of Correlated Risk has further dramatic effects on the average choice. The observed pattern of decisions in this treatment leaves less than $30 \%$ of the subjects within the risk averse domain. Interestingly, the bulk of this effect is driven by females.

The paper is organized as follows. In Section 2 and 3 we illustrate the design and procedure of our experiment, respectively. Section 4 presents our findings, while Section 5 summarizes and concludes.

\section{Experimental Design}

Our treatments are all built on the "Click" version of Bomb Risk Elicitation Task (BRET) introduced in Filippin and Crosetto (2016a). Subjects face a $10 \times 10$ square in which each numbered cell represents a box. They are told that 99 boxes are empty, while one contains a time bomb programmed to explode at the end of the task, i.e., after choices have been made. Below the field of boxes there are a "Collect" and a "Stop" button. Each time that a subject clicks on "Collect", one box is collected in numerical order. When the desired stopping point has been reached subjects press "Stop" and once the choice has been confirmed the task is over. $^{3}$ A screenshot of the task after 22 boxes have been collected is reported in Figure 1.

\footnotetext{
${ }^{3}$ In the original version of the BRET the process is automatic and subjects only need to click once to stop the collection of boxes.
} 


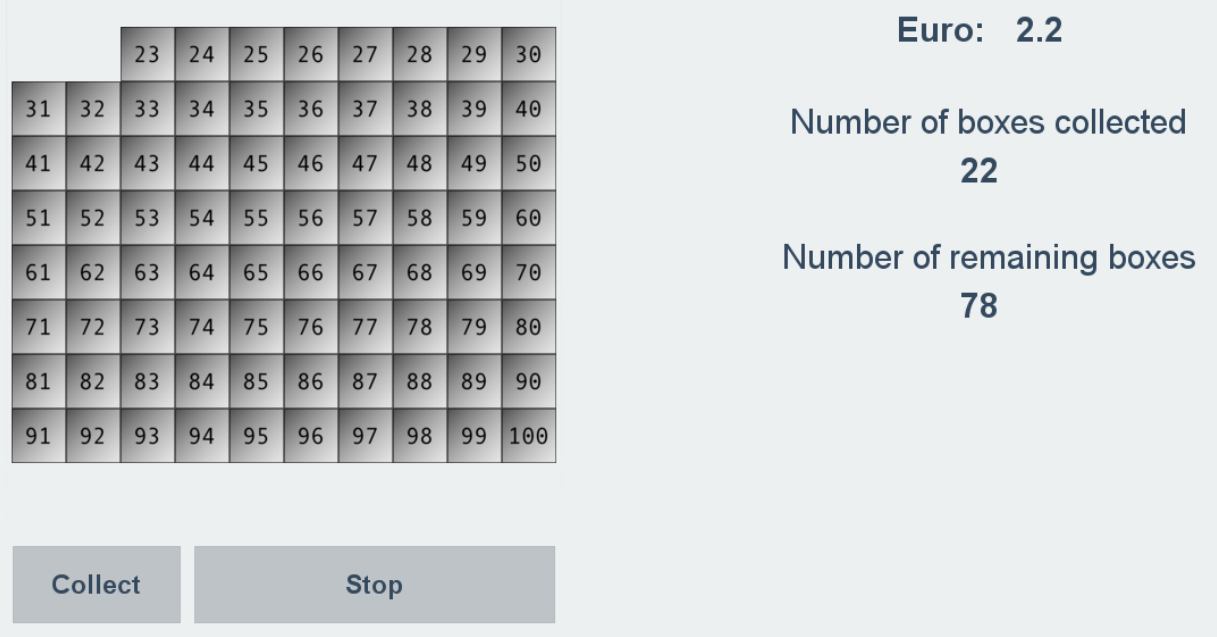

Figure 1: The BRET interface after 22 clicks

The position of the time bomb $b \in 1,100$ is randomly determined after the choice is made by means of a draw from a uniform distribution over the support $[1,100]$. Calling $k$ the number of boxes collected, if $k_{i}^{*} \geq b$ it means that subject $i$ collected the bomb, which by exploding wipes out the subject's earnings. In contrast, if $k_{i}^{*}<b$, subject $i$ leaves the minefield without the bomb and receives 10 Euro cents for each box collected. The metaphor of the time bomb allows to implement a choice in strategy method, avoiding the truncation of the data that would otherwise happen in case of a real-time notification like for instance in the Balloon Task (Lejuez et al., 2002).

Subjects' decision can be formalized as the choice of their favorite among the set $\mathcal{L}$ of 101 lotteries fully described both in terms of probabilities and outcomes by the stopping point $k \in 1,100$ :

$$
\mathcal{L}=\left\{\begin{array}{ll}
0 & \frac{k}{100} \\
0.1 k & \frac{100-k}{100}
\end{array} .\right.
$$

The parameter $k$ summarizes the trade-off between the amount of money that can be earned and the likelihood of obtaining it. The degree of measured risk aversion negatively correlates with the choice of $k$ and a risk-neutral subject should choose $k=50$.

Several features of the BRET set it apart from the tasks used in the literature on infor- 
mation cascades and conformity. In fact, the BRET operates in a context without strategic interaction and does not convey explicit informational connections. On the one hand, there is no coordination, no interaction or any form of interdependence among subjects as there is no payoff externality. On the other hand, there is no hierarchy and no temporal sequence in the decisions of the participants, who cannot communicate. The choice is completely anonymous and the payment is made privately.

The 'Click' version of the BRET potentially features a unique and unobtrusive spillover across subjects: the amount, velocity and volume of clicks that can be heard in the laboratory. The sound of others is merely suggestive of the choices (and hence of the preferences) of the other participants. In fact, subjects do not need to start the task at the same time, and they are allowed to proceed at the speed they prefer in collecting the boxes. Hearing someone clicking does not tell a subject at which stage of the task the other subject is. The clicks have no informational content toward the resolution of uncertainty either, as the position of the bomb is determined after the choices have been made. Given these considerations, the BRET looks like an inhospitable environment to observe conformist behavior, which can only rely upon hearing the others clicking, a possibility that is not even mentioned during the whole experiment.

Building on this basic task, we manipulate in a pure between-subject design $a$ ) the degree of isolation vs. exposure of the subjects to the sound of others by imposing (or not) the use of earmuffs during the task, and $b$ ) the commonality of the risk faced by performing individual or common random draws of the position of the bomb.

\subsection{Isolating subjects from the sound of others: 'Isolated'}

This treatment acts as our baseline. It replicates a situation of perfect isolation of the

subjects when making their choice as if they came individually to the laboratory. The assumption is that there is no possibility to follow others in a perfectly isolated society.

We achieve this goal introducing noise-reducing earmuffs that shield all clicking sounds coming from neighboring cubicles. Subjects are asked to wear the earmuffs right after the reading of the instructions and to keep them on for the duration of the task. Isolation from the others also requires facing idiosyncratic risk. At the end of the experiment an experimenter walks individually to each cubicle with two ten-sided dice in order to determine the 
position of the bomb. ${ }^{4}$ The experimenter rolls the dice to determine the position of the bomb separately and privately for each participant.

\subsection{Hearing others: 'Idiosyncratic Risk'}

In the Idiosyncratic Risk treatment we do not make use of earmuffs, so that participants can hear the other subjects clicking. Given a session attendance of about 30 subjects, the amount of clicking was noticeable and could be distinctly heard all over the laboratory. ${ }^{5}$

As already mentioned, the cloud of clicks represents a signal of the behavior of others with almost no informational content. In fact, subjects do not need to start at the same time and they can proceed at the speed they prefer. Nevertheless we cannot exclude that subjects attribute an informative content to the signal. If this is the case we can plausibly assume that the silence of not clicking attracts less attention than the sound of clicking, and therefore that subjects overestimate the choice of the other participants, with potential cumulative effects. Therefore, in presence of an intense and long cloud of clicks a conformist behavior should translate into a choice of a higher $k$ with respect to the baseline.

The sound of others is also orthogonal to the outcome of the task, which is determined individually following the same procedures of the Isolated treatment. By making the same choice two subjects can at most equalize the lottery ex ante, but they can still experience opposite outcomes. As illustrated in the next section, in the 'Idiosyncratic Risk' treatment there is no space for conformism based on relative payoffs. Hence, a treatment effect on the choices must rely upon an intrinsic preference for conformity and an alleged informativeness of the signal, whereby noise is more salient than silence

\subsection{All in the same boat: 'Correlated Risk'}

In the Correlated Risk condition subjects wear no earmuffs, as in the Idiosyncratic Risk treatment, but the resolution of uncertainty is common. The position of the bomb is drawn once and it applies to each and every subject in the session. In order to enhance transparency, we use a slightly different randomization device in this treatment. Subjects are asked to stand up so that they can see the front of the laboratory, where the experimenter's desk is

\footnotetext{
${ }^{4}$ One ten-sided die features printed numbers from 00 to 90 , in intervals of 10 , and is used to determine the tens; the other has sides ranging from 0 to 9 , and is used to determine the units. 100 was obtained by the result $00-0$.

${ }^{5}$ As explained in Section 3 two sessions of this treatment took place in a different lab, accommodating 18 subjects. The noise was lower, but the lab was about half the size. Results of the extra sessions are indistinguishable from the earlier ones.
} 
located. A chip is then drawn from an urn containing 100 chips, numbered from 1 to 100, and shown to the subjects. To further enhance accountability and transparency the subjects are told that they can stay at the end of the experiment to verify that the urn actually contains all the 100 chips and no other. ${ }^{6}$

This manipulation maintains the independence of the individual payoffs from the choices of any other participant, differently from what happens for instance in Thöni and Gächter (2015) and Bardsley and Sausgruber (2005). Absent any kind of interaction based on strategies, the common resolution of uncertainty might introduce a scope for conformist behavior based on social preferences. As long as subjects care about relative payoffs, they can reduce the differences by making similar choices. Both inequality concerns (Fehr and Schmidt, 1999) and social comparison considerations - for instance using the perceived choice of the others as reference point, (as summarized in Trautmann and Vieider, 2012), can rationalize the choice of a higher $k$ than the optimal one of a self-interested expected utility maximizer.

Assuming that the cloud of clicks can be mapped onto the choice of a representative opponent $k_{-i}$, Figure 2 illustrates the effect that the manipulation in the resolution of uncertainty should have for a risk neutral but loss averse player that uses the perceived choice of others as reference point. For $k_{-i}<50$ the prediction is similar in both treatments. However, if clicks matter more than silence, a risk neutral player when clicking in the interval $k \in[0 ; 50]$ perceives that the representative opponent is still collecting boxes. In other words, the signal $k_{-i}<50$ should not be perceived and if this is the case the subject does not lower his stopping point. Once the individual optimum $(k=50)$ is reached, the sound of others represents $k_{-i}>50$. The predicted behavior of the risk-neutral player then changes in the two conditions, following the sound of others (i.e. a riskier choice of the representative opponent) in the 'Correlated Risk' but not in the 'Idiosyncratic Risk' treatment.

Inequality aversion and social comparison share the key feature that the disutility of earning less than the other participants is larger than the utility of earning more. Hence, the underlying mechanism is very similar and in what follows we explain it using a social comparison framework only, i.e. clicks represent a reference point and subjects weight social losses more than equivalent gains. Denoting by $b$ the realization of the random draw and rescaling the choices $k$ from 0 to 1 for simplicity of exposition, in the 'Correlated Risk' there

\footnotetext{
${ }^{6}$ Evidence that changing the randomization device does not affect results abounds in economics (Charness and Gneezy, 2010; Filippin and Crosetto, 2016a).
} 


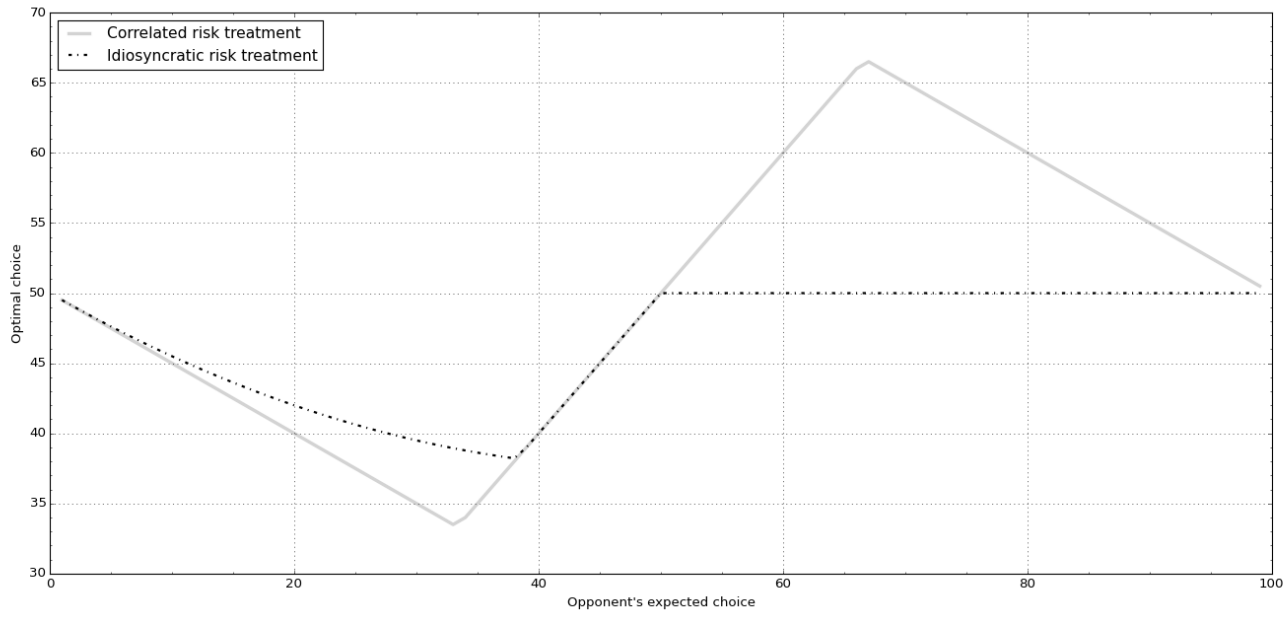

Note: the graph plots the optimal choice of a risk neutral agent characterized by a loss aversion parameter $\lambda=2$ and by a reference point corresponding to the (perceived) choice of the opponent. The best reply function is virtually identical to what would be obtained with inequality aversion preferences à la Fehr and Schmidt (1999) with $\beta=0.5$ and $\alpha=0.25$.

Figure 2: Effect of concerns for perceived relative payoffs

are only three possible outcomes:

1. $b>k_{-i}>k_{i}$ : both players win with probability $1-k_{-i}$, but $i$ earns a lower amount

2. $k_{-i} \geq b>k_{i}$ : only player $i$ wins a positive amount, with probability $k_{-i}-k_{i}$,

3. $k_{-i}>k_{i} \geq b$ : both players earn zero with probability $k_{i}$

while the worst outcome (only player $-i$ wins) cannot occur as long as $k_{-i}>k_{i}$. Social losses arising when winning a positive but lower amount have the strongest effect. Therefore, social comparison induces the subject to follow a more risk seeking choice. ${ }^{7}$

In the 'Idiosyncratic Risk' treatment the resolution of uncertainty is made individually, and therefore all the four outcomes are always possible. In this case, the strongest role is played by the worst outcome in which only player $-i$ wins, something that is possible even when making the same choice. By conforming to $k_{-i}>k_{i}$ the subject would increase the probability of this event to occur, and this effect prevails over the social loss when both players win. As a result, in 'Idiosyncratic Risk' there is no role for social comparison as a driver of conformist behavior, while this is the case in Correlated Risk.

\footnotetext{
${ }^{7}$ Note that also a more risk averse choice $k_{-i}<k_{i}$ should be followed in order to avoid the worse outcome in which only the opponent wins. However, if noise prevails over silence such an effect should not be observed.
} 


\section{Procedures}

The experiment was run from April 2012 to June 2016 in two laboratories in Jena, Germany. ${ }^{8}$

We implemented a one-shot, pure between-subjects design. The sample included mainly students from the Friedrich Schiller University Jena, Germany. 90 subjects took part to the Isolated, 92 to Idiosyncratic risk, and 89 to Correlated Risk treatments, for a total of 271 subjects distributed over 10 sessions, each lasting about half an hour. Four subjects, one in Isolated, one in Idiosyncratic Risk and two in Correlated Risk submitted weakly dominated choices, i.e., stopped at 0 or 100 boxes, and were excluded from the analysis.

The experiment was computerized. The experimental software was programmed in Python (van Rossum, 1995). The experiment was incentivized. Earnings in the task (obtained on top of the show-up fee of 2.5 Euro) ranged from 0 to 7.8 Euro, with an average of 2.06 Euro. $^{9}$

Upon entering the lab, subjects were randomly assigned to a computer. In the Isolated treatment subjects found noise-reducing earmuffs on their desk. Instructions were displayed on the screen and read aloud. ${ }^{10}$ After all questions had been individually addressed, subjects in the Isolated treatment were asked to wear the earmuffs. Then, all subjects went through a trial period in order familiarize themselves with the task. At the end of the trial period, however, there was no draw of the position of the bomb in order to avoid any carryover effect in the incentivized task. The paying task was then played one-shot.

Subjects were then asked to fill a questionnaire containing demographic information (age, gender), the SOEP risk question asking to report on a $0-10$ scale: "How do you see yourself: are you generally a person who is fully prepared to take risks or do you try to avoid taking risks?" (Wagner et al., 2007), and a self-reported measure of the perceived task difficulty on a Likert scale from 0 (easy) to 10 (hard).

Just before leaving the lab the subjects were paid in cash individually and privately, according to their choices and the position of the bomb, determined following the procedures

\footnotetext{
${ }^{8}$ Most of the study was carried out in the laboratory of the Max Planck Institute (MPI) of Economics. Two sessions of the Idiosyncratic treatment took place in the laboratory of the Friedrich Schiller University, since the MPI laboratory has been discontinued. The two labs are within 300 meters of each other and share the same subject pool.

${ }^{9}$ The average is lower than what would be implied by risk neutrality ( 2.5 Euro) because draws were largely unlucky in the Correlated Risk treatment, leading to a mass (63 out of 80) of zero payoffs in this treatment.

${ }^{10}$ The English translation of the original German instructions is available in Appendix A.
} 
explained in Section 2.

\section{Results}

Results are summarized in Table 1 and show a strong influence of the (inferred) behavior of others on subjects' choices. We observe an inclination to conform to the (perceived) choice of others already in the Idiosyncratic risk treatment, in which the only external reference is the sound of the clicks in the lab. While in the Isolated treatment the average choice is virtually identical to the original results in the BRET (Crosetto and Filippin, 2013), subjects collect more boxes when exposed to the sound of others.

\begin{tabular}{llccc}
\hline & \multicolumn{2}{c}{ Stopping point } & \multicolumn{2}{c}{ SOEP } \\
Treatment & mean & st.dev & mean & st.dev \\
\hline Isolated & 47.13 & 12.53 & 4.88 & 1.95 \\
Idiosyncratic Risk & 50.25 & 10.57 & 5.03 & 1.85 \\
Correlated Risk & 56.54 & 15.29 & 5.64 & 1.90 \\
\hline
\end{tabular}

Table 1: Number of boxes collected and SOEP self-reported risk attitudes, by treatment

In the Idiosyncratic Risk treatment the difference as compared to the baseline condition is weakly significant (Mann-Whitney, p-value $=0.068)$ and with a small effect size (Cohen's $d$ $=0.270) .{ }^{11}$ Nevertheless, this result is rather surprising, since in the Idiosyncratic Risk treatment hearing the sound of others is the only thing that subjects share. Subjects display a slight inclination to spontaneously conform to the behavior of the other participants when simply exposed to an unobtrusive signal that does not convey any objective information about precise choices. Furthermore, such an effect is observed in a setting in which subjects do not interact and there is no material incentive to conform. Their payoff does not depend on the choices of the other players, and the individual randomization device allows similar choices to map onto opposite outcomes. In such an inhospitable environment, a lower degree of risk aversion can be rationalized either by an intrinsic preference to conform or by some heuristics (e.g. I am not sure about what to do and I follow what others are doing) together with an alleged informativeness of the click signal.

\footnotetext{
${ }^{11}$ Since the influence of the sound of clicks is likely to violate the assumption of independence of observations, the Mann Whitney tests reported have been bootstrapped with stratification at the session level. The reported effect sizes are virtually identical to the bootstrapped ones.
} 
There is instead no doubt about the effect of the sound of others when a small commonality is introduced in the laboratory. Sharing the same resolution of uncertainty boosts the shift towards risk loving. Choices in the Correlated Risk treatment are significantly different both from Idiosyncratic Risk (Mann-Whitney, $\mathrm{p}$-value $=0.003$, with a medium effect size, Cohen's $d=0.482$ ) and a fortiori from Isolated (Mann-Whitney, p-value $<0.001$, with a larger effect size, Cohen's $d=0.677)$. The overall effect is impressive, with the average choice in the Correlated Risk treatment (56.5) falling well into the risk loving domain. To give an intuition of the magnitude of the effect, the observed pattern of decisions implies that less than $30 \%$ of the subjects are risk averse, a result that up to our knowledge is never observed using incentivized risk elicitation tasks in the lab. ${ }^{12}$ The difference in the behavior can be appreciated looking at Figure 3, which reports kernel density estimations of the distribution of choices in the three treatments. Note, in particular, the considerably thicker density of choices in the right tail of the distribution in the Correlated Risk treatment. ${ }^{13}$

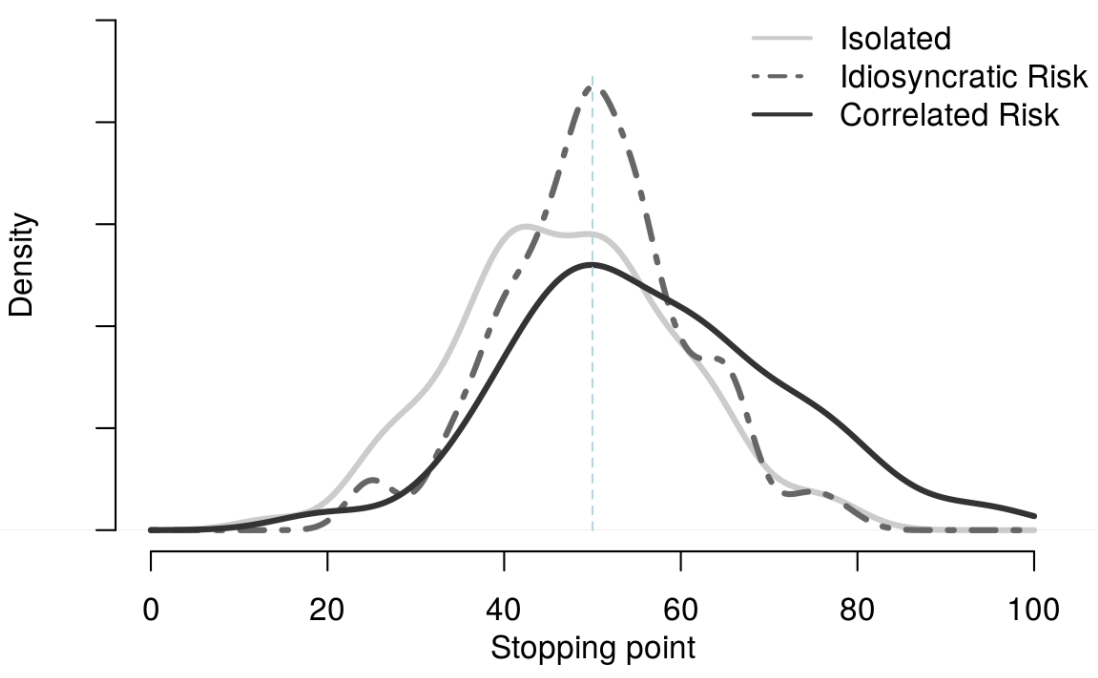

Figure 3: Kernel density estimation of choices, by treatment

\footnotetext{
${ }^{12}$ For instance, the fraction of risk averse subjects in the dataset of Holt and Laury replications presented by Filippin and Crosetto (2016b) is above $70 \%$.

${ }^{13}$ Choices are significantly different in distribution over all possible pairwise combination of treatments (Kolmogorov-Smirnov test of equality of distribution functions, bootstrapped standard errors, all p-values $\leq 0.02)$.
} 
Everything else being the same, the conformist behavior gets magnified by the common resolution of uncertainty. This result is noteworthy, in our opinion, in an environment that preserves the independence of the individual payoffs from the choices of the other participants. ${ }^{14}$ What happens in the Correlated Risk treatment is simply that payoffs are potentially correlated, since subjects can reduce ex post differences by making similar choices. Both inequality aversion and social comparison predict in our framework that subjects who dislike lower earnings proportionally more than higher earnings of the same size should conform to a more risk seeking choice of the other participants. The results in the Correlated Risk treatment strongly underline the role of social comparisons as a magnifier of conformist behavior.

Table 1 shows that subjects in the Correlated Risk also report a more risk seeking behavior in the SOEP answers. Despite the random assignment to the treatments, this evidence suggests that the results above might at least in part be explained by different risk attitudes. Indeed, equality in median of SOEP answers across the three treatments is rejected (Kruskal-Wallis p-value $=0.0164)$, because Correlated Risk differs from both Idiosyncratic Risk (Mann-Whitney, p-value $=0.015$ ) and Isolated (Mann-Whitney, p-value $=0.007)$. In principle, the choices made in the BRET may be salient when answering the SOEP question, but we tend to exclude this possibility for three reasons. First, the performance in the BRET is not known to the subjects when answering the SOEP because the position of the bomb is drawn after the questionnaire is filled. What subjects know is simply their choice in the BRET. Second, Crosetto and Filippin (2013) implement a similar protocol and find no significant correlation in a larger dataset. Third, in our data the number of boxes collected correlates positively and significantly with the self-reported SOEP measure in Isolated $(r=0.327, \mathrm{p}$-value $=0.002)$ and in Idiosyncratic Risk $(r=0.447$, p-value $<0.001)$, but not in Correlated Risk $(r=0.103, \mathrm{p}$-value $=0.343)$. Since we do not have conclusive evidence excluding that subjects in the Correlated Risk treatment may be characterized by a higher risk tolerance, in Table 2 we conservatively report the results of a multivariate regression analysis that controls for this possibility.

Column 1 includes a dummy for each treatment (Isolated omitted) plus controls for self reported risk aversion (SOEP), perceived complexity of the task, age and gender. The vari-

\footnotetext{
${ }^{14}$ This feature distinguishes our setting from the risk shift literature in which lottery choices are performed by a group (for instance after open discussion as in Shupp and Williams (2008)) and the group members receive the same payoff.
} 


\begin{tabular}{|c|c|c|c|c|c|c|}
\hline \multirow[b]{2}{*}{ Constant } & \multicolumn{3}{|c|}{$\begin{array}{c}\text { (1) } \\
\text { Stopping point }\end{array}$} & \multicolumn{3}{|c|}{$\begin{array}{c}(2) \\
\text { Stopping point } \\
\end{array}$} \\
\hline & 56.76 & $* *$ & $(2.31)$ & 51.61 & $* *$ & $(2.12)$ \\
\hline Idiosynchratic Risk & 2.991 & & $(1.47)$ & 1.674 & & $(0.61)$ \\
\hline Correlated Risk & 7.901 & $* * *$ & $(3.62)$ & -0.302 & & $(-0.10)$ \\
\hline SOEP & 1.867 & $* * *$ & $(4.24)$ & 2.015 & $* * *$ & $(4.68)$ \\
\hline female & 3.138 & * & (1.95) & -2.084 & & $(-0.79)$ \\
\hline age & -1.155 & & $(-0.86)$ & -0.737 & & $(-0.54)$ \\
\hline$a g e^{2}$ & 0.0299 & & $(1.25)$ & 0.0216 & & $(0.88)$ \\
\hline complexity & 0.115 & & $(0.36)$ & 0.244 & & $(0.79)$ \\
\hline Nsubjects & -0.352 & & $(-0.57)$ & -0.282 & & $(-0.47)$ \\
\hline Smaller lab & -5.876 & & $(-0.74)$ & -4.900 & & $(-0.64)$ \\
\hline Idiosyncratic Risk $\times$ female & & & & 1.986 & & $(0.60)$ \\
\hline Correlated Risk $\times$ female & & & & 13.62 & $* * *$ & $(3.40)$ \\
\hline$N$ & & 267 & & & 267 & \\
\hline
\end{tabular}

Table 2: Multivariate regression analysis

ables Nsubjects and Smaller lab proxy the perceived volume of clicks in the room. ${ }^{15}$ The SOEP coefficient is sizable and highly significant, but it does not explain the core result. In fact, the coefficient of the Correlated Risk dummy indicates that the effect of the sound of others under common resolution of uncertainty survives intact the introduction of the battery of controls. In contrast, the Idiosyncratic Risk dummy remains positive but does not reach traditional significance levels. Among the other control variables, also the female dummy turns out to be significant. This result is rather surprising given the gender literature in experimental contexts. First, gender differences in risk preferences are found in some risk elicitation tasks but not all, and not in the BRET, see Filippin and Crosetto (2016b). Second, when differences emerge they have the opposite sign, because females take less risks than males, not more (Charness and Gneezy, 2012; Croson and Gneezy, 2009).

In order to explain the seemingly higher level of risk taking by women in our experiment

\footnotetext{
${ }^{15}$ Since we ran the experiment in two different labs, one roughly half the size of the others, we control both for the number of subjects, as a proxy of the volume of clicks, and for the size of the room, as a proxy of the dispersion of sound. None of these are significant. Results do not change excluding the observations gathered in the smaller lab.
} 
Column 2 interacts the treatment dummies with gender. It turns out that the result of Column 1 is not linked to risk attitudes, but rather to a disproportional effect of conformism in presence of correlated risks. Note in fact that the coefficient of the female dummy is no more significant in Column 2. Furthermore, the whole effect of conformist behavior seems to be driven by females as the coefficient of Correlated Risk drops to zero. ${ }^{16}$ The pure gender effect of conformism is controversial in the literature (see Cialdini and Trost, 1998, and references therein). Women tend to be more susceptible to social influence than men, but mainly in face-to-face public interactions or in group pressure situations involving surveillance (Eagly and Carli, 1981), and the size of the gender effect is usually small. In our experiment there is no surveillance, but the cloud of clicks might act as a group pressure device on women in the Correlated Risk treatment.

\section{Conclusion}

This paper contributes to the literature showing that a conformist behavior occurs in an inhospitable environment in which the choices of others are not presented to the subjects, eliminating any kind of demand effect. We expose subjects to the "Click" version of the Bomb Risk Elicitation Task (BRET), in which the only way to spontaneously infer what the other participants are doing is the mass and volume of clicks that can be heard in the laboratory. This is at best a fuzzy signal, which does not convey any objective information about precise choices. Furthermore, individual payoffs are maintained independent from the choices of the other participants throughout the whole experiment.

A weak tendency to conform emerges already in a situation in which the resolution of uncertainty is made at the individual level, allowing similar choices to map onto opposite outcomes. Simply letting subjects hear the clicks of others induces a less risk averse behavior as compared to the acoustically isolated baseline condition. Under the assumption that noise is more salient than silence, the cloud of clicks tells subjects that the other participants are still collecting boxes. By conforming to the behavior of others, subjects go on collecting boxes and this translates into an average more risk seeking behavior. Conformist behavior is strengthened when subjects face a common resolution of uncertainty that allows a correlation in the choices to map onto a correlation in payoffs.

\footnotetext{
${ }^{16}$ The fact that only a subgroup of subjects makes a different choice may rationalize why the variance increases in the Correlated treatment (see Table 1).
} 
Our results have both theoretical and methodological implications. On the theoretical side, we show that conformism affects decisions even in contexts in which it would ex-ante be deemed unlikely. The effect in the treatment with individual resolution of uncertainty can be rationalized either by an intrinsic preference to conform or by some heuristics of the kind 'when in doubt, do as the others do,' together with an alleged informativeness of the signal. The large effect observed under a common resolution of uncertainty supports instead the role played by social preference considerations. Results are consistent with theories of inequality aversion (Fehr and Schmidt, 1999) and social reference point (Trautmann and Vieider, 2012). In our framework both models predict that subjects who dislike lower earnings proportionally more than higher earnings of the same size should conform to a more risk seeking choice of the other participants (i.e. follow the cloud of clicks) only under a common resolution of uncertainty. We interpret this result as evidence that the inclination to conform interacts with social preference considerations. A multivariate analysis displays that such a conformism in presence of correlated risks is entirely driven by women, while there are no gender differences in risk attitudes.

On the methodological side, our results warn that hearing the other subjects might create a confound in the experimental results. While not relevant in all experimental settings, the presence of bandwagon effects due to sounds might affect results in experiments characterized by quickly repeated choices, particularly if the timing of choices is crucial, such as Dutch auctions, or in the continuous-time versions of strategic games that have recently been gaining popularity (Bigoni et al., 2015; Friedman and Oprea, 2012).

\section{References}

Anderson, L., Holt, C., 1997. Information cascades in the laboratory. American Economic Review $87(5), 847-62$.

Asch, S., 1951. Groups, leadership and men. Carnegie Press, Pittsburgh, PA, Ch. Effects of group pressure on the modification and distorsion of judgments, pp. 177-190.

Asch, S., 1952. Social psychology. Prentice Hall, Englewood Cliffs, NJ.

Asch, S. E., 1955. Opinions and social pressure. Scientific American 193, 3535.

Asch, S. E., 1956. Studies of independence and conformity. a minority of one against a unanimous majority. Psychological Monographs 70(9), 1-70. 
Banerjee, A. V., 1992. A Simple Model of Herd Behavior. Quarterly Journal of Economics 107 (3), 797-817.

Bardsley, N., Sausgruber, R., 2005. Conformity and reciprocity in public good provision. Journal of Economic Psychology 26 (5), 664-681.

Bernheim, B. D., 1994. A theory of conformity. Journal of Political Economy 102 (5), 841-877.

Bicchieri, C., Xiao, E., 2009. Do the right thing: but only if others do so. Journal of Behavioral Decision Making 22 (2), 191-208.

Bigoni, M., Casari, M., Skrzypacz, A., Spagnolo, G., 2015. Time horizon and cooperation in continuous time. Econometrica 83 (2), 587-616.

Bikhchandani, S., Hirshleifer, D., Welch, I., 1992. A Theory of Fads, Fashion, Custom, and Cultural Change in Informational Cascades. Journal of Political Economy 100 (5), 992-1026.

Bikhchandani, S., Hirshleifer, D., Welch, I., 1998. Learning from the behavior of others: Conformity, fads, and informational cascades. Journal of Economic Perspectives 12 (3), 151-170.

Charness, G., Gneezy, U., 01 2010. Portfolio Choice And Risk Attitudes: An Experiment. Economic Inquiry 48 (1), 133-146.

Charness, G., Gneezy, U., 2012. Strong Evidence for Gender Differences in Risk Taking. Journal of Economic Behavior \& Organization 83 (1), 50-58.

Cialdini, R. B., Trost, M. R., 1998. Social influence: Social norms, conformity, and compliance. In: Gilbert, D. T., Fiske, S. T., Lindzey, G. (Eds.), Handbook of social psychology, 4th Edition. Vol. 2. New York: McGraw-Hill, p. 151192.

Corazzini, L., Greiner, B., October 2007. Herding, social preferences and (non-)conformity. Economics Letters 97 (1), 74-80.

Crosetto, P., Filippin, A., August 2013. The 'bomb' risk elicitation task. Journal of Risk and Uncertainty $47(1), 31-65$.

Croson, R., Gneezy, U., June 2009. Gender Differences in Preferences. Journal of Economic Literature 47 (2), 448-74.

Eagly, A. H., Carli, L., 1981. Sex of researchers and sex-typed communications as determinants of sex differences in influenceability: A meta-analysis of social influence studies. Psychological Bulletin 90, 1-20. 
Eagly, A. H., Crowley, M., 1986. Gender and helping behavior: A meta-analytic review of the social psychological literature. Psychological Bulletin, 283-308.

Fehr, E., Schmidt, K. M., 1999. A theory of fairness, competition, and cooperation. Quarterly journal of Economics, 817-868.

Filippin, A., Crosetto, P., 2016a. Click'n'roll: No evidence of illusion of control. De Economist 164 (3), 281-295.

Filippin, A., Crosetto, P., 2016b. A reconsideration of gender differences in risk attitudes. Management Science forthcming.

Fosgaard, T. R., Hansen, L. G., Piovesan, M., 2013. Separating Will from Grace: An experiment on conformity and awareness in cheating. Journal of Economic Behavior \& Organization 93 (C), 279 284.

Friedman, D., Oprea, R., 2012. A continuous dilemma. American Economic Review 102 (1), 337-63.

Goeree, J., Yariv, L., 2015. Conformity in the lab. Journal of the Economic Science Association, 1-22.

Haney, C., Banks, C., Zimbardo, P., 1973a. A study of prisoners and guards in a simulated prison. Naval Research Reviews 30, 4-17.

Haney, C., Banks, C., Zimbardo, P., 1973b. Interpersonal Dynamics in a Simulated Prison. International Journal of Criminology and Penology 1, 69-97.

Hung, A. A., Plott, C. R., 2001. Information cascades: Replication and an extension to majority rule and conformity-rewarding institutions. American Economic Review 91 (5), 1508-1520.

Lejuez, C., Read, J., Kahler, C., Richards, J., Ramsey, S., Stuart, G., Strong, D., Brown, R., 2002. Evaluation of a behavioral measure of risk taking: The Balloon Analogue Risk Task (BART). Journal of Experimental Psychology: Applied 8 (2), 75.

Quiamzade, A., L'Huillier, J.-P., 2009. Herding by attribution of privileged information. Journal of Behavioral Decision Making 22 (1), 1-19.

Shupp, R. S., Williams, A. W., 2008. Risk preference differentials of small groups and individuals. The Economic Journal 118 (525), 258-283.

Thöni, C., Gächter, S., 2015. Peer effects and social preferences in voluntary cooperation: A theoretical and experimental analysis. Journal of Economic Psychology 48 (0), 72 - 88.

Trautmann, S. T., Vieider, F. M., 2012. Social Influences on Risk Attitudes: Applications in Economics. Springer Netherlands, Dordrecht, pp. 575-600. 
van Rossum, G., 1995. Python reference manual. CWI Report CS-R9525.

Wagner, G. G., Frick, J. R., Schupp, J., 2007. The german socio-economic panel study (soep): Scope, evolution and enhancements. Schmollers Jahrbuch 1271 (1), 139-169.

Ziegelmeyer, A., Koessler, F., Bracht, J., Winter, E., 2010. Fragility of information cascades: anexperimental study usingelicited beliefs. Experimental Economics 13 (2), 121-145. 


\section{Appendix A. Instructions}

\section{Welcome screen}

You are about to participate in an experiment in which following the instructions carefully, making good decisions, and with a bit of luck, you can earn money. Different participants may earn different amounts according to their choices. For your participation in the experiment you will earn an additional show-up fee of 2.5 Euro. All the monetary values during the experiment are expressed in Euro cents.

\section{Procedure Screen}

The experiment consists of 4 stages in the following order:

1. An Instruction Stage that we are currently going through.

2. A Decision Stage, in which you will make decisions and answer questions relevant to your final payoff.

3. A Questionnaire, in which you will be asked a few questions not related to your final payoff.

4. A Feedback Stage, in which your earnings from the experiment will be privately determined. You will not be given any feedback on the monetary outcome of your decisions before the Feedback Stage.

\section{Task instructions screen}

On your screen you will see a field composed of boxes numbered 1 through 100 . Your task is to decide on the number of boxes to collect out of 100 such boxes. You earn 10 Euro cents for each box collected. At any moment you can see the amount earned up to that point. Such earnings are only potential, however, because exactly one of these 100 boxes contains a time bomb, that if collected destroys all the boxes collected. You do not know the bombs location. You only know that it is equally likely to be in any of the 100 boxes. Moreover, even if you collect the time bomb, you will not know it until the end of the experiment.

In order to collect each box you have to click once the Collect' button that you will see on the screen. Boxes are collected starting from the top left corner and following the numerical order until you decide to stop collecting and hit the Stop button when the number corresponds to how many boxes you want to collect. Once collected, each box disappears 
from the screen. At the end of the experiment, after answering some questions and filling out a short questionnaire, the number of the box containing the bomb will be randomly determined.

[Earmuffs, Idiosyncratic Risk] An experimenter will come at your desk with two 10-sided dice. One will be used to determine the tens, the other the units. After letting you check that the dice are regular, the experimenter will roll them to determine the position of the bomb between 1 and 100 .

[Correlated Risk] At the end of the experiment we will ask one of you to come to the front and randomly determine the position of the bomb, by drawing a chip from an urn containing 100 chips, numbered from 1 to 100.

If the number of the box in which the bomb is located is higher than the number of boxes you collected, you do not collect the bomb and you earn 10 Euro cents for each collected box. If the number of the box in which the bomb is located is lower than or equal to the number of boxes you collected, you do collect the bomb and you earn zero.

We will now start with a trial round. After the trial round, we will begin the payoffrelevant task.

Earmuffs screen

[Earmuffs] Now if everything is clear please wear the earmuffs you see on your desk. You will be notified in another screen later when you have to remove them.

\section{Decision screen}

Please choose the number of boxes to collect.

In order to collect each box you have to click once the Collect' button.

Boxes are collected starting from the top left corner and following the numerical order. When the number corresponds to how many boxes you want to collect hit the Stop button. Then confirm your choice by clicking on Confirm. 